\title{
Comportamiento de las Paredes de Dominio Ferroeléctricas en una Nanoesfera de Titanato de Plomo
}

\author{
Andrade-Landeta, Julio ${ }^{1}$ iD ; Lascano-Lascano, Luis ${ }^{2, ~ * ~ i c ~}$ \\ ${ }^{1}$ Colegio Politécnico, Universidad San Francisco de Quito, Ecuador \\ ${ }_{2}^{2}$ Departamento de Física, Escuela Politécnica Nacional, Quito - Ecuador
}

\begin{abstract}
Resumen: El objetivo del presente trabajo fue estudiar el comportamiento de las paredes de dominio ferroeléctricas en una nanoesfera de titanato de plomo bajo diferentes condiciones térmicas, eléctricas y mecánicas. Para ello se ha hecho uso de la teoría fenomenológica de Ginzburg-Landau y para obtener el estado de equilibrio se utilizaron principios variacionales; las ecuaciones que aparecen en el desarrollo se resolvieron analíticamente. Los resultados obtenidos proveen un perfil de la polarización dentro de las paredes de dominio $180^{\circ}$ de la nanoesfera de titanato de plomo, así como el espesor de dicha pared en función de la temperatura y para distintas condiciones de la nanoesfera. Se observa que, con el aumento de la temperatura, el perfil de la polarización se reduce y el espesor de la pared crece al acercarse a cierta temperatura; todo lo cual permitiría sintonizar la temperatura de transición ferroeléctrica mediante el control del tamaño de la nanoestructura, de la presencia de cargas libres y de la aplicación de esfuerzos mecánicos.
\end{abstract}

Palabras clave: Pared de dominio, transición ferroeléctrica, titanato de plomo, polarización.

\section{Behavior of Ferroelectrics Domain Walls in a Lead Titanate Nanosphere}

\begin{abstract}
The objective of the present work was to study the behavior of the ferroelectric domain walls in a lead titanate nanosphere under different thermal, electrical and mechanical conditions. For this, the phenomenological theory of Ginzburg-Landau has been used and variational principles were used to obtain the state of equilibrium; the equations that appear in development were solved analytically. The results obtained provide a profile of the polarization within the $180^{\circ}$ domain walls of the lead titanate nanosphere, as well as the thickness of said wall as a function of temperature and for different conditions of the nanosphere. It is observed that, with the increase in temperature, the polarization profile decreases and the wall thickness increases as it approaches a certain temperature; all of which would allow tuning the ferroelectric transition temperature by controlling the size of the nanostructure, the presence of free charges and the application of mechanical stresses.
\end{abstract}

Keywords: Domain wall, ferroelectric transition, lead titanate, polarization.

\section{INTRODUCCIÓN}

Un dominio en una fase ferroica, como puede ser un material ferroeléctrico o uno ferromagnético, está definido como la región que contiene una configuración espacial local ordenada de un parámetro de orden, el cual puede ser la polarización o la magnetización, respectivamente. Los diferentes dominios son distinguibles por su orientación espacial del parámetro de orden.

La mayor parte de la investigación actual sobre aplicaciones de materiales ferroicos se enfoca en los límites entre dominios vecinos, también conocidos como paredes de dominio, por sus notables propiedades funcionales (Prando, 2017).
Existen diversas investigaciones acerca del uso de las paredes de dominio como elementos activos en dispositivos electrónicos, y muy particularmente en memorias no volátiles (Foerster et al., 2016; Salje, 2010; Li et al., 2005; Catalan et al., 2012; Grimley et al., 2018; Chopra et al., 2000); sin embargo, a pesar de varios avances en la manipulación de las paredes de dominio ferroeléctrico y en el control de su conductividad, los principios del funcionamiento en dispositivos de memoria aún no están completamente definidos (Prando, 2017).

El presente trabajo tuvo como objetivo modelar y estudiar teóricamente el comportamiento de las paredes de dominio de $180^{\circ}$ en una nanoesfera ferroeléctrica de titanato de plomo (PbTiO3), dando así continuidad al trabajo de Andrade y

*luis.lascano@epn.edu.ec

Recibido: 18/05/2020

Aceptado: 22/09/2020

Publicado: 30/11/2020

10.33333/rp.vol46n2.04

CC BY 4.0 
Lascano (2017), donde se estudiaron los efectos que produce la disminución de tamaño de la nanoesfera, al estar sometida a la acción simultánea de campos de despolarización y de esfuerzos mecánicos. Aquí se buscó conocer el comportamiento de las paredes de dominio en la nanoesfera de titanato de plomo bajo la acción de los mismos campos.

Para la realización de este estudio se han tomado en cuenta varios trabajos sobre paredes de dominio (Lee et al., 2009; Pöykkö y Chadi, 1999; Meyer y Vanderbilt, 2002; Behera et al., 2011) realizados mediante primeros principios y con la teoría del funcional de la densidad, en los cuales se ha calculado el perfil de la polarización de pared de dominio para ciertos arreglos de celdas unitarias vecinas, tomando en cuenta el desplazamiento de los átomos dentro de la celda unitaria con parámetros macroscópicos fijos tales como la temperatura $T=0 K$ (Pöykkö y Chadi, 1999), es decir, dentro de un escenario estático de la fase ferroeléctrica. En cambio, aquí buscamos conocer el comportamiento de las paredes de dominio en función de la temperatura y bajo diversas condiciones de la nanoesfera de PT.

El conocimiento del comportamiento de las paredes de dominio de una nanoestructura ferroeléctrica puede utilizarse para predecir la respuesta piezoeléctrica de este tipo de cristales; como también puede ser de mucha ayuda en el diseño de cristales nanométricos para uso en dispositivos de alta piezoelectricidad, donde se aprovechan técnicas de ingeniería de configuración de dominio ( $\mathrm{Li}$ y Chen, 2006; Wada et al., 2007; Bell et al., 2020; Lipatov et al., 2019; Jiang et al.,2018).

Los resultados obtenidos en este estudio muestran la influencia que tienen la temperatura, el tamaño de partícula y los esfuerzos mecánicos sobre el perfil de la polarización en la pared de dominio $180^{\circ}$, y sobre el espesor de dicha pared en una nanoesfera de titanato de plomo.

Estos resultados pueden ser útiles al estudiar la inversión de polaridad de los dominios ferroeléctricos bajo la acción de campos eléctricos externos en condiciones termo-mecánicas de funcionamiento, con miras a utilizar estos materiales en dispositivos tales como sensores, actuadores, memorias ferroeléctricas de acceso aleatorio. Además, son importantes los estudios a nivel nanométrico porque permiten la construcción de una alta densidad de memoria guardada en circuitos nano electrónicos (Faraji et al., 2017; Rojac y Damjanovic, 2017; Kumar et al., 2020; Scott et al., 2016; Chai et al., 2020).

El estudio aquí desarrollado pretende avanzar en la comprensión de las propiedades ferroeléctricas de un cristal a escala nanométrica.

\section{METODOLOGÍA}

La presente investigación de carácter teórico estudia el comportamiento de las paredes de dominio de una nanoestructura de titanato de plomo bajo diferentes condiciones. El sistema a estudiar consiste de las paredes de dominio $180^{\circ}$ de una nanoesfera ferroeléctrica de titanato de plomo (PT), rodeada por una capa de cargas espaciales en su superficie y sometida a la acción simultánea del campo de despolarización y de esfuerzos mecánicos.

El modelo teórico propuesto se fundamenta en la teoría fenomenológica de Gizburg-Landau para cristales ferroeléctricos, tomando como parámetro de orden a la polarización del cristal. Entonces se plantea la densidad volumétrica de energía del sistema tomando en cuenta las magnitudes siguientes: la energía de un cristal mono axial simple ferroeléctrico bajo ausencia de campos eléctricos externos, la energía de despolarización generada por la formación de los dominios de $180^{\circ}$ en la nanoesfera rodeada por una capa superficial de cargas libres, la energía provocada por la presencia de esfuerzos mecánicos y la energía de pared de dominio.

Posteriormente, se realiza un cálculo de tipo variacional buscando la configuración de equilibrio estable del sistema y, de allí, la polarización y el espesor de la pared de dominio. En ese desarrollo se llega a una ecuación diferencial compleja cuya solución se la encuentra mediante el uso de integrales elípticas de tercer orden. Así se obtiene una solución para el perfil de polarización de la pared de dominio y para el espesor de dicha pared. Finalmente se hace un análisis cuantitativo del comportamiento de la pared de dominio en función de parámetros variables tales como la temperatura, el diámetro de la partícula, el grosor de la capa de cargas libres que cubren la nanoesfera y los esfuerzos mecánicos, mediante el uso del lenguaje de programación Python.

\section{Densidad de energía libre}

El análisis parte con la determinación de la densidad de energía libre de Ginzburg-Landau-Devonshire de la nanoesfera de PT, a la cual se añaden la densidad de energía de despolarización, la densidad de energía de paredes de dominio y la densidad de energía electro-elástica.

La densidad volumétrica de energía libre, $F_{P}$, de un cristal ferroeléctrico en ausencia de campos eléctricos externos se expresa como serie de potencias pares de la polarización $P$ (Rabe et al., 2007):

$$
F_{P}=\frac{1}{2} \alpha P^{2}+\frac{1}{4} \beta P^{4}+\frac{1}{6} \zeta P^{6}
$$

donde $\alpha=\alpha_{0}\left(T-T_{0 \infty}\right)$ depende a la vez de la temperatura T de la nanoesfera y de la temperatura de transición T0 $\infty$ del PT. Allí $\alpha_{0}, \beta$ y $\zeta$ son coeficientes cuyos valores pueden obtenerse desde experimentos, o pueden calcularse por métodos de primeros principios (Lallart, 2011).

A la anterior densidad de energía se añade la densidad de energía de despolarización, $F_{d}$, debida al campo eléctrico de despolarización, la cual representa la energía eléctrica necesaria para la formación de los dominios de $180^{\circ}$ en la nanoesfera (Andrade y Lascano, 2017):

$$
F_{d}=\epsilon^{*} \frac{t d}{D^{2}}\left[1-e^{-2 \pi\left(\frac{D}{d}\right)}\right] P^{2}
$$


donde $\epsilon^{*}=\frac{4}{\epsilon_{0} \pi^{3}}, D$ es el diámetro de la nanoesfera de PT, $d$ es el grosor de cada dominio de $180^{\circ}$. Además, allí se considera una nanoesfera cubierta por una capa de grosor $t$ de cargas eléctricas espaciales.

Asimismo, también debe añadirse la densidad de energía $F_{\sigma}$ correspondiente al efecto de esfuerzos mecánicos $\sigma$ aplicados sobre la nanoesfera de PT, los cuales pueden también originarse en la transición desde una fase paraeléctrica cúbica a una fase ferroeléctrica tetragonal del PT. Ya que la fase ferroeléctrica del PT es tetragonal, su polarización está determinada únicamente por la componente $P_{3}$ del campo de polarización $\vec{P}=\left(0,0, P_{3}\right)$, (Sun et al., 2010), es decir, $P=P_{3}$. Además, al contrario de lo realizado en el trabajo de Cao y Cross (1991), aquí se toma en cuenta la condición hidrostática para los esfuerzos mecánicos, esto es: $\sigma 1=\sigma 2=\sigma 3=\sigma, \sigma 4=$ $\sigma 5=\sigma 6=0$. Por lo tanto, la densidad de energía $F_{\sigma}$ es (Andrade y Lascano, 2017):

$$
F_{\sigma}=-\frac{3}{2}\left(s_{11}+s_{12}\right) \sigma^{2}-\left(Q_{11}+2 Q_{12}\right) \sigma P^{2}
$$

donde $\operatorname{los} Q_{i j}$ son los coeficientes electroestrictivos.

De este modo, sumando las Ecuaciones (1), (2) y (3), obtenemos la energía libre por unidad de volumen, notada como $F_{1}$, de una nanoesfera ferroeléctrica con una estructura de dominios de $180^{\circ}$, su valor es:

$$
\begin{aligned}
& F_{1}=\frac{1}{2} \alpha P^{2}+\frac{1}{4} \beta P^{4}+\frac{1}{6} \zeta P^{6}+\epsilon^{*} \frac{t d}{D^{2}}\left[1-e^{-2 \pi\left(\frac{D}{d}\right)}\right] P^{2}- \\
& -\frac{3}{2}\left(s_{11}+s_{12}\right) \sigma^{2}-\left(Q_{11}+2 Q_{12}\right) \sigma P^{2}
\end{aligned}
$$

Todos los valores de los coeficientes que intervienen en la densidad de energía $F_{1}$ de la Ecuación (4) aparecen detallados en la Tabla 1.

Tabla 1. Parámetros del cristal de titanato de plomo

\begin{tabular}{cll}
\hline Parámetro & Valor & Referencia \\
\hline$T_{0 \infty}$ & $752 \mathrm{~K}$ & Zhang et al. (2008) \\
$\alpha_{0}$ & $7,6 \times 10^{5} \mathrm{C}^{-2} \mathrm{~m}^{2} \mathrm{~N}$ & Rabe et al. (2007) \\
$\beta$ & $-2,92 \times 10^{8} \mathrm{C}^{-2} \mathrm{~m}^{2} \mathrm{~N}$ & Idem \\
$\zeta$ & $1,56 \times 10^{9} \mathrm{C}^{-6} \mathrm{~m}^{10} \mathrm{~N}$ & Idem \\
$Q_{11}$ & $0,089 \mathrm{C}^{-2} \mathrm{~m}^{4}$ & Idem \\
$Q_{12}$ & $-0,026 \mathrm{C}^{-2} \mathrm{~m}^{4}$ & Idem \\
$S_{11}$ & $-2,5 \mathrm{C}^{-12} \mathrm{~m}^{2} \mathrm{~N}^{-1}$ & Idem \\
$s_{12}$ & $9,0 \mathrm{C}^{-12} \mathrm{~m}^{2} \mathrm{~N}^{-1}$ & Idem \\
\hline
\end{tabular}

Agrupando los términos de P 2 en la Ecuación (4) se obtiene:

$$
\begin{aligned}
F_{1}= & -\frac{3}{2}\left(s_{11}+s_{12}\right) \sigma^{2}+\left\{\frac{1}{2} \alpha+\epsilon^{*} \frac{t d}{D^{2}}\left[1-e^{-2 \pi\left(\frac{D}{d}\right)}\right]-\left(Q_{11}+\right.\right. \\
& \left.\left.+2 Q_{12}\right) \sigma\right\} P^{2}+\frac{1}{4} \beta P^{4}+\frac{1}{6} \zeta P^{6}
\end{aligned}
$$

Finalmente, a la anterior densidad de energía se le añade la contribución de la densidad de energía de pared de dominio, $F_{w}$, la cual depende del gradiente del campo de polarización que existe en la pared, y que representa físicamente la densidad volumétrica de energía en la dirección de mayor cambio de la polarización en el espacio, y puede expresarse así:

$$
F_{w}=\frac{1}{2} g|\nabla \boldsymbol{P}|^{2}
$$

donde $g$ es el coeficiente de energía del gradiente de polarización que depende de la naturaleza del cristal.

De este modo, añadiendo a la energía dada en (5) la densidad de energía de la relación (6), se obtiene la densidad de energía del sistema en estudio:

$$
F=-\frac{3}{2}\left(s_{11}+s_{12}\right) \sigma^{2}+\frac{1}{2} A P^{2}+\frac{1}{4} \beta P^{4}+\frac{1}{6} \zeta P^{6}+\frac{1}{2} g|\boldsymbol{\nabla} \boldsymbol{P}|^{2}
$$

donde por facilidad de análisis se ha introducido el coeficiente

$$
\frac{1}{2} A=\frac{1}{2} \alpha+\epsilon^{*} \frac{t d}{D^{2}}\left[1-e^{-2 \pi\left(\frac{D}{d}\right)}\right]-\left(Q_{11}+2 Q_{12}\right) \sigma .
$$

Además, si se limita el estudio al caso cuasi-unidimensional en el que la variación de la polarización ocurre tan solo en dirección perpendicular a la misma, y si se denomina como eje $\mathrm{X}$ a aquel de la dirección de cambio de la polarización, con el origen en el centro del dominio, entonces el gradiente de la polarización se reduce a $\nabla \mathrm{P}=\frac{\mathrm{dP}}{\mathrm{dx}}$, y la densidad volumétrica de la energía libre del sistema resulta ser:

$$
F=-\frac{3}{2}\left(s_{11}+s_{12}\right) \sigma^{2}+\frac{1}{2} A P^{2}+\frac{1}{4} \beta P^{4}+\frac{1}{6} \zeta P^{6}+\left.\frac{1}{2} g|| \frac{\mathrm{dP}}{\mathrm{dx}}\right|^{2}
$$

\section{RESULTADOS Y DISCUSIÓN}

\subsection{Los estados de equilibrio}

Una vez determinada la densidad de energía libre del sistema, el equilibrio estático del mismo se puede obtener aplicando el método variacional a dicha densidad de energía, el cual se expresa por:

$$
\frac{\partial}{\partial x}\left(\frac{\partial F}{\partial\left(\frac{d P}{d x}\right)}\right)-\frac{\partial F}{\partial P}=0
$$

Se puede considerar que los dominios del sistema constituyen fases homogéneas, es decir, que dentro de cada uno de ellos no hay variación de las magnitudes físicas, por lo que la Ecuación (9) se reduce a $\frac{\partial F}{\partial P}=0$, condición que aplicada a la Ecuación (8) equivale a:

$$
\mathrm{AP}+\beta \mathrm{P}^{3}+\zeta \mathrm{P}^{5}=0
$$

cuyas soluciones proporcionan los valores de polarización de los estados de equilibrio de los dominios, los mismos que son:

$$
P=0
$$

$\mathrm{y}$

$$
P_{0}= \pm\left[\frac{-\beta+\left(\beta^{2}-4 A \zeta\right)^{\frac{1}{2}}}{2 \zeta}\right]^{\frac{1}{2}}
$$

La solución $P=0$ corresponde a la fase cúbica paraeléctrica del sistema, y la solución (11) describe los dos estados de polarización de la fase ferroeléctrica tetragonal, es decir que la 
polarización en tal fase está dada por $\vec{P}=\left(0,0, \pm\left|P_{0}\right|\right)$. La dirección de $\vec{P}$ es perpendicular a la dirección $\mathrm{X}$ definida anteriormente. Son justamente estas dos posibilidades de orientación de la polarización, energéticamente equivalentes, las que dan lugar a la estructura de dominios de $180^{\circ} \mathrm{y}$, por consiguiente, en la aparición de una pared de dominio entre dos de ellos.

La pared de dominio es una zona de transición entre los dos valores de la polarización $\vec{P}$ dados por la Ecuación (11), y como tal es una región heterogénea del sistema. Por ello, el perfil de la polarización en la pared de dominio se puede obtener aplicando el principio variacional (9) a la densidad de energía libre dada por la Ecuación (8), lo cual conduce a la siguiente relación:

$$
g \frac{d^{2} P}{d x^{2}}-A P-\beta P^{3}-\zeta P^{5}=0
$$

Para la resolución de la Ecuación (12) dentro de la pared de dominio se ha realizado un procedimiento análogo a los realizados en los trabajos de Cao y Cross (1991) y de Falk (1983), involucrando el uso de integrales elípticas de tercera clase. Las condiciones de borde necesarias consisten en el hecho antes descrito de que la polarización a los costados de la pared toma los valores $\left(0,0, \pm\left|P_{0}\right|\right)$, las cuales además corresponden a la dirección [001]. Meyer y Vanderbilt (2002) y Torres Sancho (2009) reportan la existencia de paredes de dominio de $180^{\circ}$ paralelas a esas mismas direcciones en el PT para su fase ferroeléctrica tetragonal. Con todo ello, se obtiene la solución siguiente:

$$
P(x)=\frac{P_{0} \sinh \left(\frac{x}{\xi_{180^{\circ}}}\right)}{\left[C+\sinh ^{2}\left(\frac{x}{\xi_{180^{\circ}}}\right)\right]^{1 / 2}}
$$

donde:

$$
\begin{gathered}
\xi_{180^{\circ}}=\frac{\sqrt{g}}{P_{0}\left(\zeta P_{0}{ }^{2}+\frac{1}{2} \beta\right)^{1 / 2}} \\
C=\frac{6 \zeta P_{0}{ }^{2}+3 \beta}{4 \zeta P_{0}{ }^{2}+3 \beta}
\end{gathered}
$$

La Expresión (13), solución de la Ecuación (12), representa el perfil de la polarización a través de la pared de dominio en la fase ferroeléctrica tetragonal de la nanoesfera, mientras que la Ecuación (14) representa el espesor de la pared de dominio de $180^{\circ}$. Tanto el perfil como el espesor dependen de las condiciones en la que se encuentra la nanoesfera a través de las cantidades $A$ y $P_{0}$, y es lo que se analiza a continuación.

Para obtener resultados numéricos a partir de las soluciones antes encontradas es necesario conocer el valor del coeficiente $g$ de energía de gradiente para el PT, además de hacer uso de los valores presentados en la Tabla 1. La literatura ofrece datos provenientes indirectamente de medidas experimentales, y otros provenientes de simulaciones para sistemas de PT próximos al que se estudia en este trabajo. En el trabajo de Li et al. (2001) se dan valores experimentales de coeficientes $G_{i j}$ del gradiente de energía de pared de dominio, tales que para el sistema aquí en estudio, que es cuasi unidimensional, se tiene que $g \equiv \mathrm{G}_{11}=0,6 \mathrm{G}_{110}$, donde $\mathrm{G}_{110}$ es una constante para normalizar $\mathrm{G}_{11}$ durante la simulación y se relaciona con el espaciado de la cuadrícula en el espacio real (Choudhury et al., 2005). Con base en medidas experimentales, Li et al. (2001) manifiestan que el valor de $\mathrm{G}_{110}$ está en el intervalo comprendido entre $0,9 \times 10^{-10}$ y $6,8 \times 10^{-10} \mathrm{C}^{-2} \mathrm{~m}^{4} \mathrm{~N}$. Si bien Shin et al. (2007) mediante simulación en dinámica molecular han calculado coeficientes del gradiente de polarización con valores menores asociados a los planos (100), en el presente trabajo se ha decidido adoptar, en primer lugar, un valor experimental y, en segundo lugar, el valor menor del intervalo antes anotado, por cuanto este valor está relacionado con el estado de menor energía de pared de dominio y, por tanto, posiblemente el más probable; dicho valor es $g=0,6 \times$ $0,9 \times 10^{-10} C^{-2} m^{4} N=0,54 \times 10^{-10} C^{-2} m^{4} N$ y es el que se ha utilizado en los cálculos que aquí se presentan.

No se han encontrado estudios experimentales que muestren el perfil de la pared de dominio de $180^{\circ}$ del titanato de plomo en función de la temperatura, tamaño de partícula, y la capa de cargas eléctricas libres en su superficie; y tampoco la de grosor de pared de dominio en función de los parámetros ya mencionados a escala nanométrica. Por otro lado, existen trabajos como los de los autores Lee et al. (2009), Behera et al. (2011) y Pöykkö y Chadi (1999), donde mediante cálculos de primeros principios y de DFT se obtienen perfiles de la polarización en la pared de dominio del titanato de plomo para el cuerpo masivo, de modo que su escala en cuanto al grosor es de los amstrongs, lo cual no es compatible para realizar una comparación con el modelo aquí presentado que se refiere a una escala nanométrica.

\subsection{Polarización en la pared de dominio $180^{\circ}$}

En la Figura 1 se presenta el perfil de polarización en función de la posición dentro de la pared de dominio de la nanoesfera de $\mathrm{PbTiO} 3$ para una polarización espontánea del cuerpo masivo igual a $\left|P_{0}\right|=0,75 \mathrm{C} / \mathrm{m}^{2}$, y obtenida a partir de la Ecuación (13). Allí se observa que la polarización cambia de signo cuando cruza el centro de la pared, como era de esperarse, y se satura en los valores $P_{0}= \pm 0,75 \mathrm{C} / \mathrm{m}^{2}$. La polarización sigue el comportamiento de una función simétrica alrededor del centro de la pared de dominio.

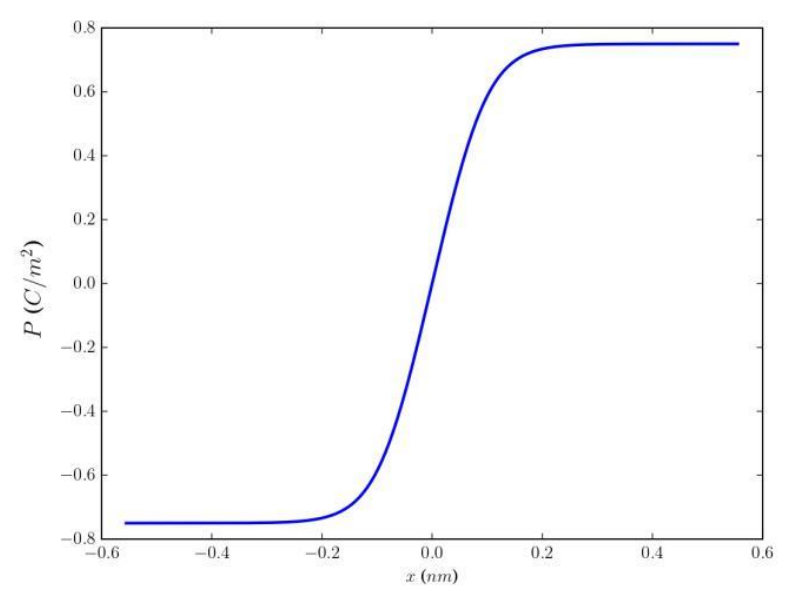

Figura 1. Polarización en función de la posición en la pared de dominio de $180^{\circ}$, para una polarización espontánea de cuerpo masivo $\mathrm{P} 0=0,75 \mathrm{C} / \mathrm{m} 2$ 
Los resultados que se muestran a continuación provienen de aplicar las Ecuaciones (13) ó (14) simultáneamente con la Ecuación (11). Esto se realiza por cuanto se quiere conocer el comportamiento de la polarización de la pared de dominio, o el grosor de pared de dominio, en función de parámetros tales como son la temperatura, el diámetro de la nanoesfera, el espesor de la capa cargas espaciales que cubre a la nanoesfera de PT y los esfuerzos mecánicos, todos los cuales se encuentran implícitamente en la Ecuación (11).

En la Figura 2 se presenta el perfil de la polarización en función de la posición dentro de la pared de dominio $180^{\circ}$ para varias temperaturas del sistema. Allí se observa que, en general, la polarización decrece notablemente cuando la temperatura se incrementa; a las temperaturas de $0 \mathrm{~K}, 298,15$ $\mathrm{K}$ y $500 \mathrm{~K}$ los perfiles de polarización son semejantes entre sí, no así para la temperatura de $636,67 \mathrm{~K}$ donde el valor de la polarización experimenta una reducción notable y el comportamiento es muy distinto al que aparece para las temperaturas antes mencionadas. Esto es, al igual de lo que sucede con la polarización espontánea en los dominios ferroeléctricos, el valor de la polarización a través de la pared de dominio se reduce drásticamente cuando la temperatura del sistema se aproxima a la de su transición ferroeléctrica.

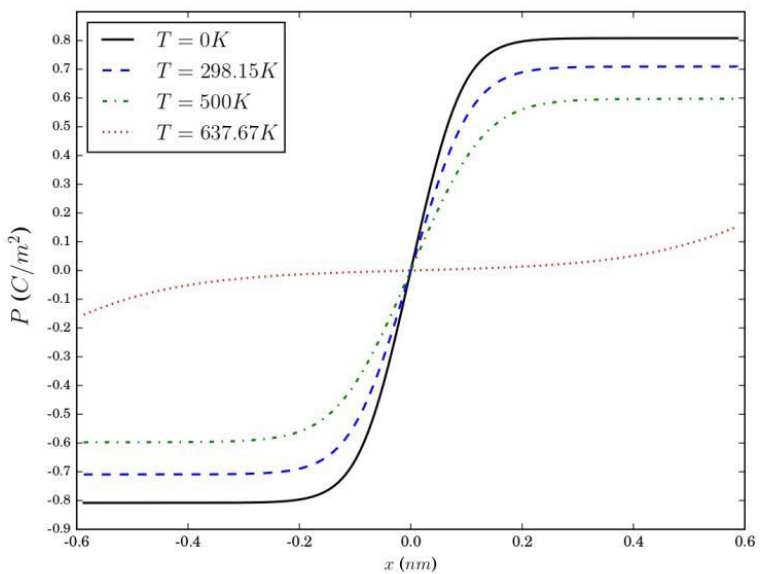

Figura 2. Polarización en función de la posición en la pared de dominio de $180^{\circ}$ de una nanoesfera de diámetro $20 \mathrm{~nm}$ a diferentes temperaturas, con configuración de 3 dominios y capa de cargas libres de espesor $\mathrm{t}=0,2 \mathrm{~nm}$

La Figura 3 presenta la polarización en función de la posición dentro de una pared de dominio $180^{\circ}$ para una nanoesfera de PT con diferentes diámetros, un espesor fijo de la capa de cargas libres en su superficie y a temperatura ambiente. Se observa que, para todas las posiciones, al disminuir el tamaño de partícula también disminuye el valor de la polarización, y esta reducción es muy pronunciada para el valor de diámetro $D=5,47 \mathrm{~nm}$ de la nanoesfera. Este valor de diámetro es el valor crítico calculado en el trabajo de Andrade y Lascano (2017) para la nanopartícula de PT, diámetro para el cual la polarización espontánea prácticamente se anula.

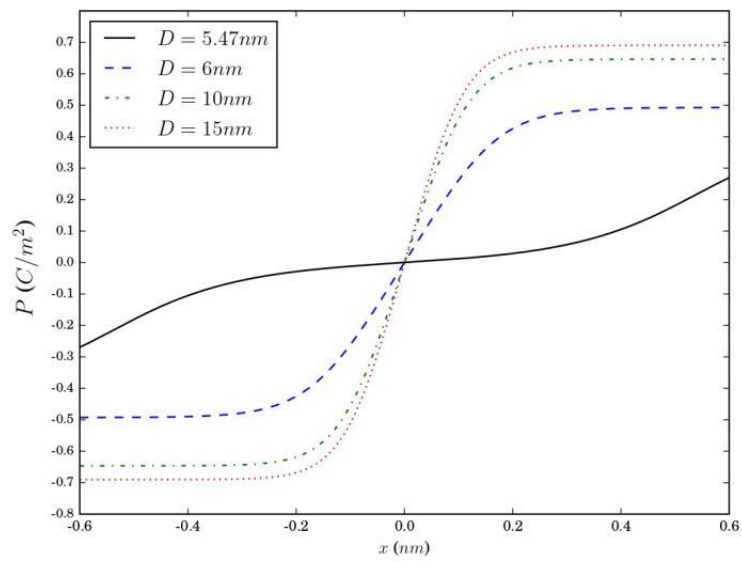

Figura 3. Polarización en función de la posición en la pared de dominio de $180^{\circ}$, para varios diámetros de la nanoesfera, con configuración de 3 dominios, capa de cargas libres de espesor $\mathrm{t}=0,2 \mathrm{~nm}$ y a temperatura ambiente $\mathrm{T}=298,15 \mathrm{~K}$

En la Figura 4 se presenta el perfil de la polarización en función de la posición dentro de una pared de dominio de $180^{\circ}$ en una nanoesfera de PT de $20 \mathrm{~nm}$ de diámetro y a temperatura ambiente, para diferentes espesores de la capa superficial de cargas libres. El aumento del grosor de la película de cargas libres sobre la superficie de la nanopartícula reduce, para todas las posiciones, el valor de la polarización en la pared de dominio. Particularmente, para el valor de $t=0,73 \mathrm{~nm}$, hay una gran distorsión del perfil de la polarización respecto al perfil que se observa para el espesor $t=0,20 \mathrm{~nm}$, lo cual expresa la fuerte influencia de la capa de cargas libres sobre el campo de despolarización dentro de la pared de dominio.

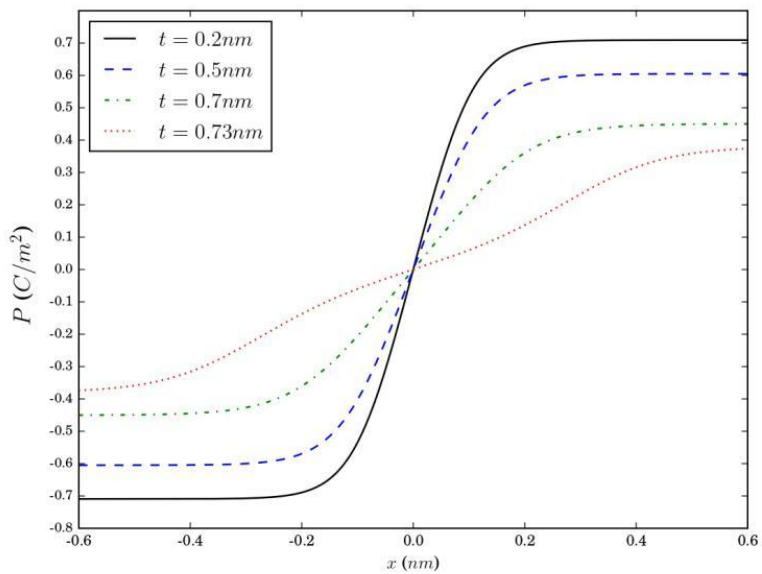

Figura 4. Polarización en función de la posición en pared de dominio de $180^{\circ}$ de una nanoesfera de diámetro $\mathrm{D}=20 \mathrm{~nm}$, para varios grosores de capa de cargas libres superficiales, con configuración de 3 dominios y a temperatura ambiente $\mathrm{T}=298,15 \mathrm{~K}$

La Figura 5 presenta la polarización en función de la posición en una pared de dominio $180^{\circ}$ para una nanoesfera de PT con $100 \mathrm{~nm}$ de diámetro y a $50 \mathrm{~K}$ de temperatura, para diferentes valores de esfuerzos mecánicos aplicados sobre la misma. Se observa que los esfuerzos de tensión (esfuerzos positivos) hacen que el perfil de polarización aumente ligeramente en relación con el perfil de polarización en ausencia de esfuerzos (línea negra). En contraste, bajo la presencia de esfuerzos de compresión (esfuerzos negativos) el perfil de polarización en la pared de dominio se reduce significativamente respecto al 
caso de la nanoesfera libre de esfuerzos; tal reducción es mayor a medida que los esfuerzos de compresión aumentan, y podría suceder que para valores elevados de esfuerzos de compresión se altere la estabilidad ferroeléctrica del sistema. Puede sugerirse que, a escala nanométrica, la aplicación de esfuerzos de compresión elevados provoca una reducción del tamaño de la nanoesfera ferroeléctrica, y con ello una amplificación del efecto de tamaño de partícula ya observado en el trabajo de Andrade y Lascano (2017).

\subsection{Espesor de pared de dominio de $180^{\circ}$}

En la Figura 6 se representa el espesor de la pared de dominio en función de la polarización espontánea de la nanoesfera de PT. Allí se observa que el espesor de pared de dominio se incrementa de manera abrupta cuando la polarización espontánea se acerca su valor crítico dado por $\mathrm{P}\left(\mathrm{T}=\mathrm{T}_{\mathrm{C}}^{-}\right)=$ $\sqrt{-\frac{\beta}{2 \zeta}}=3,053 \times 10^{-1} \mathrm{C} / \mathrm{m}^{2}$ y calculado en el trabajo de Andrade y Lascano (2017) para la nanoesfera de titanato de plomo. Aparece así un resultado peculiar: un crecimiento brusco del espesor de la pared de dominio cuando la partícula se acerca a la transición ferroeléctrica. Una posible interpretación es que este acelerado crecimiento del grosor de pared de dominio hace que la pared de dominio vaya ocupando más volumen dentro de la nanoesfera hasta cubrir todo el cristal, cuando éste se acerca a la transición ferroeléctrica. Por otro lado, para valores de polarización superiores al crítico $\mathrm{P}\left(\mathrm{T}=\mathrm{T}_{\mathrm{C}}^{-}\right)=\sqrt{-\frac{\beta}{2 \zeta}}=3,053 \times 10^{-1} \mathrm{C} / \mathrm{m}^{2}$, el grosor de pared de dominio se reduce notablemente y tiende a cero.

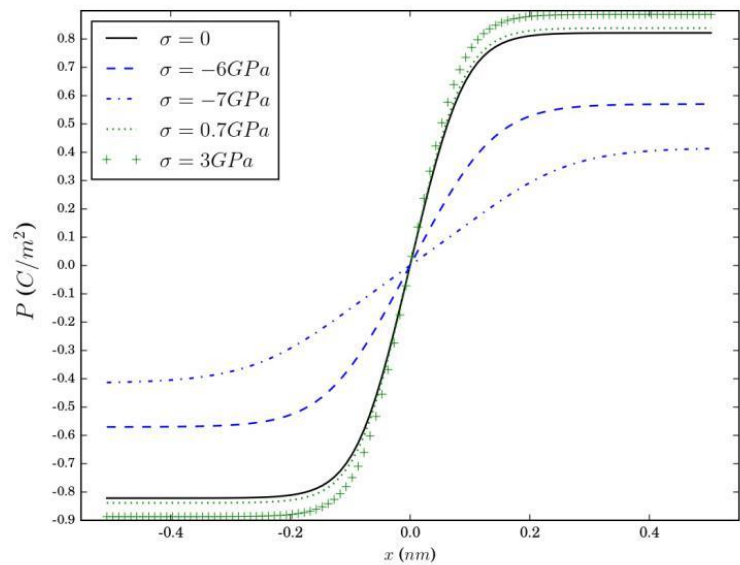

Figura 5. Polarización en función de la posición en pared de dominio $180^{\circ}$ para diferentes esfuerzos mecánicos aplicados a una nanoesfera de PT con diámetro $\mathrm{D}=100 \mathrm{~nm}$, con capa de cargas superficiales de grosor $\mathrm{t}=0,2 \mathrm{~nm}$, con configuración de 3 dominios y a la temperatura $\mathrm{T}=50 \mathrm{~K}$

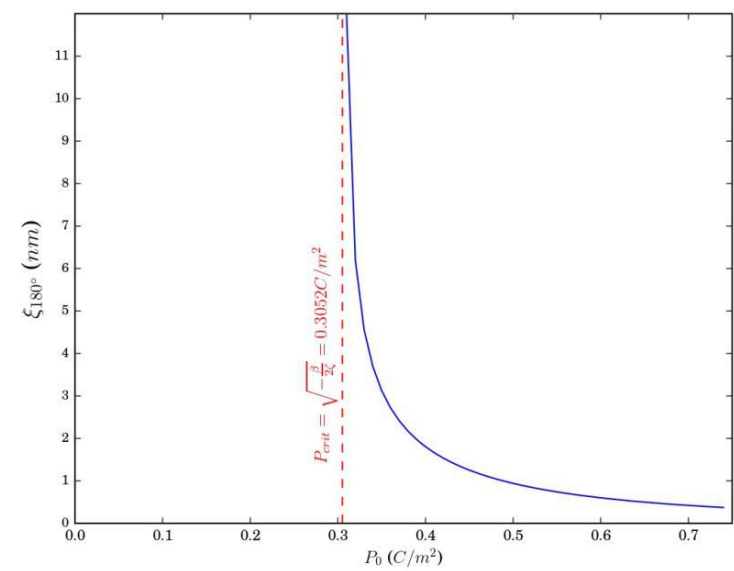

Figura 6. Espesor de la pared de dominio en función de la polarización espontánea de una nanoesfera de titanato de plomo

La Figura 7 presenta el espesor de la pared de dominio de una nanoesfera de PT en función de la temperatura y para diferentes diámetros de la nanopartícula. Se observa que, para un diámetro definido, el espesor crece con el aumento de la temperatura y lo hace de manera muy abrupta cuando la temperatura se acerca a cierto valor, el cual es diferente para cada diámetro de la partícula. Tal temperatura es la de Curie de la transición ferroeléctrica calculada en el trabajo de Andrade y Lascano (2017), y se ve que la misma se reduce con la disminución del tamaño de partícula, lo cual concuerda con el análisis que se realiza en el trabajo mencionado. Además, se observa que a $0 \mathrm{~K}$ de temperatura y para todos los diámetros de la partícula, el grosor de la pared de dominio es cercano a los $0,3 \mathrm{~nm}$.

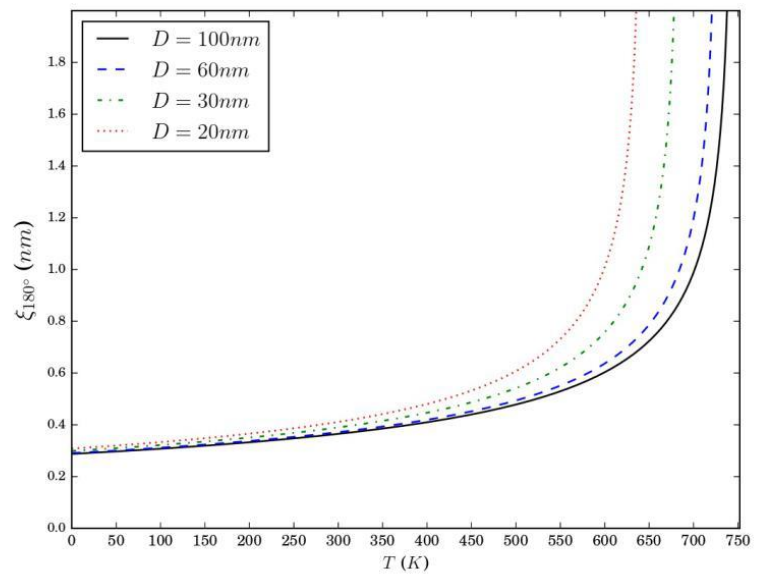

Figura 7. Espesor de la pared de dominio de nanoesfera de titanato de plomo en función de la temperatura, para diferentes diámetros de la nanoesfera, con capa de cargas superficiales de grosor de $\mathrm{t}=0,2 \mathrm{~nm}$ y con configuración de 3 dominios

En la Figura 8 se presenta el espesor de la pared de dominio en función de la temperatura de la nanoesfera de PT con $20 \mathrm{~nm}$ de diámetro y configuración de 3 dominios, para distintos valores del espesor de la capa superficial de cargas libres. Como era de esperarse, se observa el mismo aumento brusco del espesor cuando crece la temperatura; el valor de temperatura para el cual el incremento es súbito es aquel de la transición ferroeléctrica; tal valor se reduce de manera notable 
con el aumento del espesor de la capa de cargas libres superficiales, reduciéndose así la estabilidad de la fase ferroeléctrica. Además, a la temperatura de $0 \mathrm{~K}$, el valor del grosor de la pared crece con el aumento del espesor de la capa de cargas superficiales.

La Figura 9 muestra el espesor de la pared de dominio en función de la temperatura de una nanoesfera de PT con $100 \mathrm{~nm}$ de diámetro y para diferentes esfuerzos aplicados. Se han considerado 3 dominios en la nanoesfera y una capa de cargas libres superficiales con $0,2 \mathrm{~nm}$ de espesor. En general, se observa el mismo comportamiento de los dos casos anteriores en relación con el aumento de la temperatura. Y además se observa que los esfuerzos de tensión aplicados provocan un incremento de la temperatura de transición ferroeléctrica y, por el contrario, los esfuerzos de compresión reducen tal temperatura. Por otro lado, para la temperatura de $0 \mathrm{~K}$ el valor del grosor de pared de dominio crece con el aumento de la magnitud de los esfuerzos de compresión, y prácticamente no se altera con los esfuerzos de tensión.

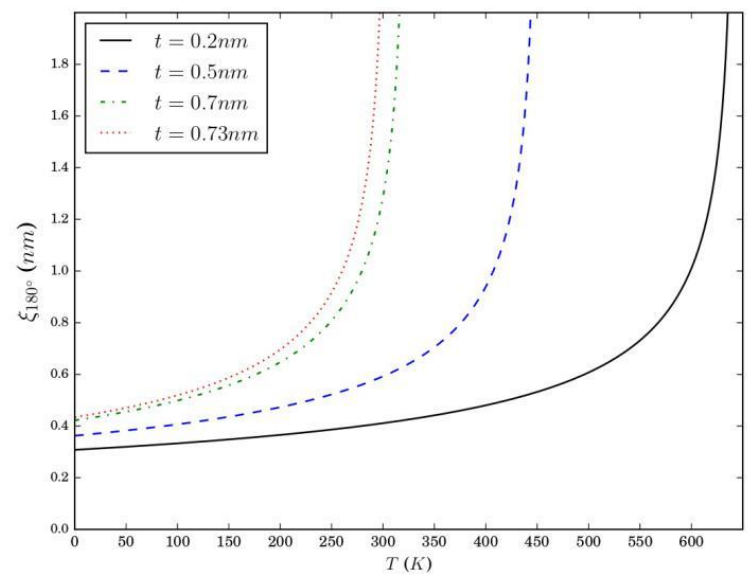

Figura 8. Espesor de la pared de dominio en función de la temperatura de nanoesfera de titanato de plomo con diámetro $\mathrm{D}=20 \mathrm{~nm}$ y configuración de 3 dominios, para diferentes grosores $t$ de la capa de cargas superficiales

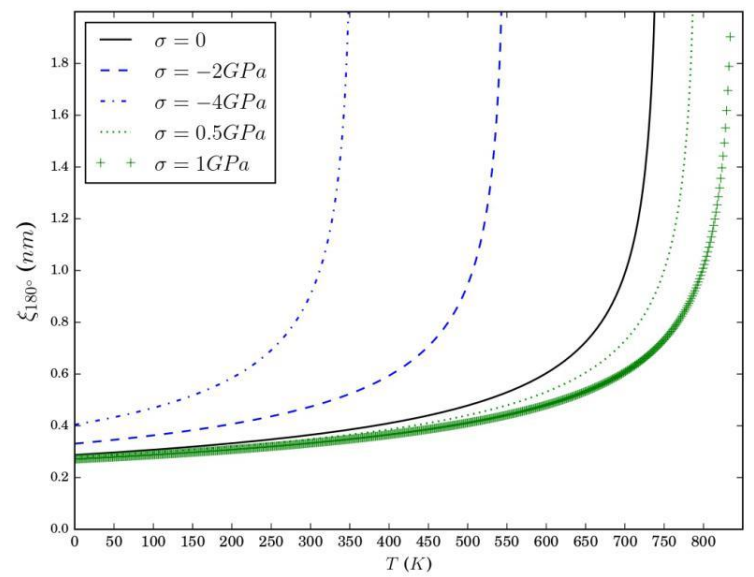

Figura 9. Espesor de la pared de dominio en función de la temperatura de nanoesfera de titanato de plomo de diámetro $\mathrm{D}=100 \mathrm{~nm}$, para diferentes esfuerzos mecánicos $\sigma$ aplicados a la nanoesfera, con configuración de 3 dominios y capa de cargas superficiales de espesor $\mathrm{t}=0,2 \mathrm{~nm}$

\section{CONCLUSIONES}

El presente modelo prevé que el perfil de la polarización de pared de dominio de $180^{\circ}$ de la nanoesfera de titanato de plomo tiende a desaparecer y a deformarse con el aumento de la temperatura, con la reducción del tamaño de la nanopartícula, con el aumento del grosor de la capa de cargas espaciales en la superficie de la misma, y con la aplicación de esfuerzos de compresión. Por el contrario, la estabilidad del carácter ferroeléctrico del sistema se ve favorecido con la presencia de esfuerzos de tensión y con el incremento del tamaño de partícula.

$\mathrm{Al}$ aumentar la temperatura y al aproximarse a la temperatura de transición ferroeléctrica (la cual depende del diámetro de la nanoesfera, del espesor de la capa de cargas espaciales y de la presencia de esfuerzos), el espesor de la pared de dominio de la nanoesfera de titanato de plomo crece de forma abrupta. La pared de dominio es una zona heterogénea de polarización y si su espesor crece súbitamente (tal que el espesor podría ocupar toda la nanoesfera) significa que la fase ferroeléctrica está tornándose inestable; además, simultáneamente la polarización misma en la pared va disminuyendo y tiende a desaparecer. Estos dos aspectos pertenecen pues al mismo fenómeno: el cristal está transitando de la fase ferroeléctrica a la paraeléctrica.

Los resultados aquí encontrados muestran principalmente cómo la temperatura de transición ferroeléctrica de una nanoestructura puede ser sintonizable controlando parámetros tales como: tamaño de la nanoestructura, presencia de cargas espaciales, y aplicación de esfuerzos de tensión y/o compresión.

\section{REFERENCIAS}

Andrade, J. y Lascano, L. (2017). Comportamiento ferroeléctrico de una nanoesfera de titanato de plomo debido a campos de despolarización y a esfuerzos mecánicos. Boletín de la Sociedad Española de Cerámica y Vidrio, $56 \quad$ (1), 19-28. http://dx.doi.org/10.1016/j.bsecv.2016.07.001

Behera, R.K., Lee, C.-W., Lee, D., Morozovska, A.N., Sinnott, S.B., Asthagiri, A., Gopalan, V. y Phillpot, S.R. (2011). Structure and energetics of $180^{\circ}$ domain walls in $\mathrm{PbTiO} 3$ by density functional theory. Journal of Physics: Condensed Matter, 23(17), 175902. https://doi.org/10.1088/0953-8984/23/17/175902

Bell, A.J., Shepley, P.M., y Li, Y. (2020). Domain Wall Contributions to Piezoelectricity in Relaxor-Lead Titanate Single Crystals. Acta Materialia, 195, 292303. https://doi.org/10.1016/j.actamat.2020.05.034

Cao, W. y Cross, L.E. (1991). Theory of tetragonal twin structures in ferroelectric perovskites with a firstorder phase transition. Phys. Rev. B, 44(1), 5-12. https://doi.org/10.1103/PhysRevB.44.5 
Catalan, G., Seidel, J., Ramesh, R., y Scott, J.F. (2012). Domain wall nanoelectronics. Reviews of Modern Physics, 84(1), 119-156. https://doi.org/10.1103/RevModPhys.84.119

Chai, X., Jiang, J., Zhang, Q., Hou, X., Meng, F., Wang, J., Gu, L., Zhang D.W. y Jiang, A.Q. (2020). Nonvolatile ferroelectric field-effect transistors. Nature Communications, 11(1), 1-9. https://doi.org/10.1038/s41467-020-16623-9

Chopra, H.D., Ji, C. y Kokorin, V.V. (2000). Magnetic-fieldinduced twin boundary motion in magnetic shapememory alloys. Phys. Rev. B, 61(22), R14913. https://doi.org/10.1103/PhysRevB.61.R14913

Choudhury, S., Li, Y., y Chen, L-Q. (2005). A phase diagram for epitaxial PbZr1-xTixO3 thin films at the bulk morphotropic boundary composition. J. Am. Ceram. Soc., $\quad 88(6), \quad 1669 \quad-\quad 1672$. https://doi.org/10.1111/j.1551-2916.2005.00319.x

Falk, F. (1983). Ginzburg-Landau theory of static domain walls in shape-memory alloys. Zeitschrift für Physik B Condensed Matter, 51(2), 177-185. https://doi.org/10.1007/BF01308772

Faraji, N., Yan, Z., y Seidel, J. (2017). Electrical conduction at domain walls in lead titanate (PbTiO3) single crystals. Applied Physics Letters, 110(21), 213108. https://doi.org/10.1063/1.4983642

Foerster, M., Boulle, O., Esefelder, S., Mattheis, R., Kläui M. (2016). Domain Wall Memory Device. In: Xu Y., Awschalom D., Nitta J. (eds) Handbook of Spintronics. Springer, Dordrecht. https://doi.org/10.1007/978-94-007-6892-5_48

Grimley, E.D., Schenk, T., Mikolajick, T., Schroeder, U. y LeBeau, J.M. (2018). Atomic structure of domain and interphase boundaries in ferroelectric $\mathrm{HfO} 2$. Advances Materials Interfaces, 5(5), 1701258. https://doi.org/10.1002/admi.201701258

Jiang, J., Bai, Z. L., Chen, Z.H., He, L., Zhang, D.W., Zhang, Q.H., Shi, J.A, Park, M.H., Scott, J.F., Hwang C.S. y Jiang, A. Q. (2018). Temporary formation of highly conducting domain walls for non-destructive read-out of ferroelectric domain-wall resistance switching memories. Nature materials, 17(1), 49-56. https://doi.org/10.1038/nmat5028

Kumar, A., Guy, J.G., Zhang, L., Chen, J., Gregg, J.M. y Scott, J.F. (2020). Nanodomain patterns in ultra-tetragonal lead titanate (PbTiO3). Applied Physics Letters, 116(18), 182903. https://doi.org/10.1063/5.0007148

Lallart, M. (Ed). (2011). Ferroelectrics - Applications. Rijeka, Croatia: Intech Open.

Lee, D., Behera, R.K., Wu, P., Xu, H., Li, Y.L., Sinnott, S.B., Phillpot, S.R., Chen, L.Q. y Gopalan, V. (2009).
Mixed Bloch-N'eel-Ising character of $180^{\circ}$ ferroelectric domain walls. Phys. Rev. B, (80), 060102.

https://doi.org/10.1103/PhysRevB.80.060102

Li, J., Rogan, R., Üstündag, E. y Bhattacharya, K. (2005). Domain switching in polycrystalline ferroelectric ceramics. Nature Materials. 4 (10), 776-781. https://doi.org/10.1038/nmat1485

Li, Y.L., Hu, S., Liu, Z.K., y Chen, L.Q. (2001). Phase-field model of domain structures in ferroelectric thin films. Applied Physics Letters, 78(24), 3878-3880. https://doi.org/10.1063/1.1377855

Li, Y.L. y Chen, L.Q. (2006). Temperature-strain phase diagram for $\mathrm{BaTiO} 3$ thin films. Applied physics letters, $\quad 88(7), \quad 072905$. https://doi.org/10.1063/1.2172744

Lipatov, A., Li, T., Vorobeva, N.S., Sinitskii, A. y Gruverman, A. (2019). Nanodomain engineering for programmable ferroelectric devices. Nano Letters, 19(5), 3194-3198. https://doi.org/10.1021/acs.nanolett.9b00673

Meyer, B. y Vanderbilt, D. (2002). Ab initio study of ferroelectric domain walls in PbTiO3. Phys. Rev. B, 65(10) 104111. https://doi.org/10.1103/PhysRevB.65.104111

Pöykkö, S. y Chadi, D.J. (1999). Ab initio study of $180^{\circ}$ domain wall energy and structure in PbTiO3. Applied Physics Letters, 75(18), 2830-2832. https://doi.org/10.1063/1.125164

Prando, G. (2017). Walls and memory. Nature Nanotech, 12, 724. https://doi.org/10.1038/nnano.2017.166

Rabe, K.M., Ahn, C.H., y Triscone, J.M. (Eds), (2007). Physics of ferroelectrics: A modern perspective. Heidelberg, Germany, Springer, 368.

Rojac, T. y Damjanovic, D. (2017). Domain walls and defects in ferroelectric materials. Japanese Journal of Applied Physics, 56(10S), 10PA01. https://doi.org/10.7567/JJAP.56.10PA01

Salje, E.K. (2010). Multiferroic domain boundaries as active memory devices: Trajectories towards domain boundary engineering. Chemphyschem: European Journal of Chemical Physics and Physical Chemistry, 11(5), 940-950. https://doi.org/10.1002/cphc.200900943

Scott, J.F., Evans, D.M., Gregg, J.M. y Gruverman, A. (2016). Hydrodynamics of domain walls in ferroelectrics and multiferroics: Impact on memory devices. Applied Physics Letters, 109(4), 042901. https://doi.org/10.1063/1.4959996 
Shin, Y.-H., Grinberg, I., Chen I.-W., y Rappe, A.M. (2007). Nucleation and growth mechanism of ferroelectric domain-wall motion. Nature, 449, 881-884. https://doi.org/10.1038/nature06165

Sun, T., Wang, X., Wang, H., Zhang, X., Cheng, Z., Sun, C. y Li, L. (2010). A phenomenological model on phase transitions in nanocrystalline barium titanate ceramic. Journal of the American Ceramic Society, 93(9), 2571 - 2573. https://doi.org/10.1111/j.15512916.2010.03900.x

Torres Sancho, M. (2009). Study of ferroelectric PbTiO3 nanostructures deposited onto substrates and prepared by a novel microemulsion mediated synthesis. (PhD thesis), Instituto de Química y Materiales “Alvaro Alonso Barba” (Universidad Carlos III de Madrid), Madrid.

Wada, S., Muraishi, T., Yokoh, K., Yako, K., Kamemoto, H. y Tsurumi, T. (2007). Domain wall engineering in lead-free piezoelectric crystals. Ferroelectrics, 355(1), 37-49. https://doi.org/10.1080/00150190701515881

Zhang, S., Li, H., y Li, M. (2008). Size-dependent piezoelectric coefficient d33 of $\mathrm{PbTiO} 3$ nanoparticles. Materials Letters. 62(16), 2438-2440. https://doi.org/10.1016/j.matlet.2007.12.015

\section{BIOGRAFÍAS}

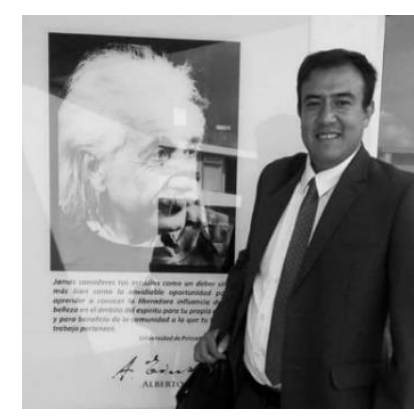

Julio César Andrade Landeta. Nació en Santo Domingo de los Tsáchilas, Ecuador en 1987. Físico de la Escuela Politécnica Nacional del Ecuador, 2014. Su área de investigación es la de transiciones de fase en cristales ferroeléctricos. En la actualidad se encuentra realizando sus estudios de

Posgrado en la Maestría de Física de la Universidad San Francisco de Quito. https://orcid.org/0000-0003-0176-1373

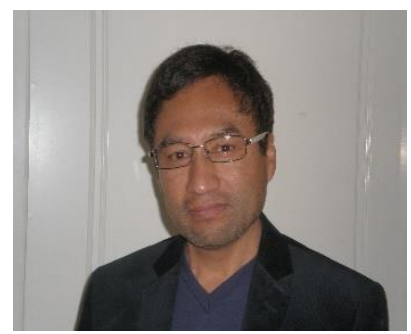

Luis Rodrigo Lascano Lascano. Doctorado en Física de Materiales, Universidad Autónoma de Madrid e Instituto de Cerámica y Vidrio, España. Físico, Escuela Politécnica Nacional (EPN), Quito. Profesor Principal del Departamento de Física de la EPN. Miembro del Grupo de Investigación de Materiales Electrónicos y Magnéticos de dicho departamento. Las líneas de investigación que desarrolla son: Estudios teórico y experimental de Materiales Electrocerámicos Ferroeléctricos, y estudios teóricos en Óptica no lineal. ORCID: 0000-00017588-0194. 
